\title{
Utilization of objective structured clinical examination as an assessment strategy for undergraduate nursing students
}

Prof MN Sibiya (Corresponding author)

Department of Nursing

Durban University of Technology

Email: nokuthulas@dut.ac.za

Prof M Lekhuleni

Department of Nursing Science

University of Limpopo

Email: Masamo.Lekhuleni@ul.ac.za

\begin{abstract}
The Objective Structured Clinical Examination (OSCE) has been widely and increasingly used since its inception in 1975. It was specifically focused on assessing the clinical skills of medical students. Since then the OSCE has been adapted for use in other health professional curricula, particularly nursing. At undergraduate level, three factors that indicate that OSCE is best used for the assessment of psychomotor skills are, firstly, that undergraduate nurses operate towards the novice end of the novice-expert continuum. Secondly, nurses must be sufficiently competent to practise their profession safely prior to clinical placement and, finally, the difficulties of replicating a real-world clinical environment in an examination context need to be acknowledged. This article, therefore, provides an overview of the utilisation of OSCE as an assessment tool for undergraduate nursing students. Different approaches to OSCE, preparation and planning for OSCE, scoring rubric, quality assurance strategies, advantage and disadvantages of this assessment approach are discussed.
\end{abstract}

Key words: Assessment, Competence, Objective Structured Clinical Examination (OSCE), Undergraduate nursing students 


\section{Introduction}

The assessment of clinical competence is an essential requirement of health professional education, with standardised procedures ensuring objectivity and maximising the reliability of assessments. The Objective Structured Clinical Examination (OSCE) is a popular tool for assessing clinical competence in nursing (Harden et al., 1975). It was first introduced in medical education in 1975 by Ronald Harden at the University of Dundee in Scotland. OSCE is a multiple station examination in which all learners need to perform a number of clinical tasks within a specified time-frame. The interaction of the assessor and the learners is guided by established rules and instructions. OSCE also entails an assessment where learners demonstrate their competence under a variety of simulated conditions. Since the 1970s, OSCE has been a valuable strategy for assessing clinical skills and knowledge acquisition in medical students (Rushforth, 2007).

OSCE is usually performed in simulation laboratories as it is meant to assess psychomotor, cognitive and affective skills. Several clinical skills' stations are created, consisting of various clinical practices that nursing students should demonstrate competence in (Meyer \& van Niekerk, 2008). Higher education nurse curricula currently utilize OSCE as a means of summative assessment to test a variety of clinical skills learnt over a period of time, and depending on the nature of the programme, this could be at the end of a teaching block or end of the year as a final examination (Clarke, Rainey \& Traynor, 2011). In OSCE, students are expected to demonstrate competence in a variety of simulated situations. OSCE integrates knowledge and skills, enabling components of clinical competence and performance to be identified and assessed under standardised conditions. This allows for a large number of students to be assessed simultaneously (Clarke, Rainey \& Traynor, 2011).

Although OSCE was initially invented in the United Kingdom, medical schools across the western world have increasingly adopted the assessment strategy. In the 1990s, its use across North America, Canada, Australia and other Western countries has increased (Rushforth, 2007).

\section{Rationale and aim of the article}


Effective nursing assessment strategies are required to ensure high-quality teaching and learning and to further the role of the nursing profession, the primary aim of which is to safeguard the public. Due to a lack of consensus on a precise definition for competence, deciding on an appropriate strategy to assess clinical competence has long been central to nursing and midwifery education (Smith et al., 2012). The concept of assessing the integration of skills in undergraduate nursing students contravenes Benner's (1984) novice to expert taxonomy which describes the development of competence. Benner (1982) defines nursing competence as being able to perform tasks with desirable outcomes under the diverse circumstances of the real world and defines the performance of undergraduate nursing students as being at the novice level. He describes the progression of nurses as a continuum from novice to expert with competence placed firmly in the centre of this development (Benner, 1984).

OSCE is now used within Schools of Nursing to assess both the theoretical and practical (but mainly practical) aspects of students' competence. Although much of the research on OSCE has been conducted in medical education (Khattab \& Rawlings, 2001; Rushforth, 2007), there is increasing support for OSCE within nursing education. This article therefore, provides an overview of the utilisation of OSCE as an assessment tool for undergraduate nursing students.

\section{Different approaches to OSCE}

The original OSCE, as described by Harden et al. (1975), involved a series of 16-20 skills' stations, with each station taking approximately 5 minutes to complete. The original OSCE was specifically focused on assessing the clinical skills of medical students. The term Objective Structured Practical Examination (OSPE) was derived from the OSCE in 1975 when the word 'practical' sometimes replaced 'clinical' (Harden \& Gleeson, 1979). While the OSCE focuses on assessing clinical competence, the OSPE is designed to assess competence in performing a practical skill outside the clinical context (Frantz et al., 2013). Rushforth (2007) identifies four different approaches to nursing OSCE; namely, (a) multistation OSCE, (b) scenario-based OSCE, (c) top-to-toe assessment, and (d) simulated assessment with random allocation.

Multi-station OSCE consists of a series of time-limited clinical tasks through which all students have to perform in a consecutive series of stations. At each station, the student is 
faced with a task or problem. Tasks might include test interpretation, history-taking, physical examination, patient education or other nursing activities. Students are observed by examiners whose interaction with the students is carefully regulated, usually being limited to providing instructions. Students are observed and evaluated as they go through a series of stations in which they interview, examine and/or manage standardised patients who present with some type of health problem. Standardised patients may be laypersons who are trained to simulate a specific set of symptoms (Kurz et al., 2009). Findings of a number of research studies reveal that the use of standardised patients influences student satisfaction (Kurz et al., 2009; Turner, \& Dankoski, 2008; Ebbert \& Connors, 2004; Gibbons et al., 2002). The use of actual patients presents several barriers to objective evaluations. Real patients can present with a set of symptoms that may vary from student to student and in these variations end up not matching the student's skills level. Real patients may be too ill to interact with students. Medical and legal issues can also become a barrier. Standardised patients allow for iterative actions for several students over time and a controlled level of difficulty (Kurz et al., 2009).

Since the advent of the OSCE, the format has undergone major revisions and adaptations, the most common being the use of longer duration, fewer assessment stations and an increased focus on total patient consultation (Khattab \& Rawlings, 2001; Ward \& Barratt, 2005). These modified OSCEs, sometimes referred to as Objective Structured Clinical Assessments (OSCA), have been favourably appraised for providing the potential for a more holistic approach to the assessment of clinical competence (Ward \& Willis, 2006; Rushforth, 2007). OSCA is sometimes referred to as scenario-based OSCE, consisting of one or two 'long' OSCE stations.

Top-to-toe assessment entails students performing a full examination of a patient, systematically working from head to toe across all body systems. In simulated assessment the top-to-toe assessment may be distributed in stations that follow each other so that the student is not examining one patient for 20 minutes, but examining five systems in five stations in that 20 minutes. For example, in the first station the student may be asked to examine and report on the skin, while in the very following station asked to examine and report on the upper respiratory tract. This means that all students are assessed on the same scenarios/systems. 
Several authors have expressed their concerns that OSCE undermines holism (Bujack, McMillan, Dwyer \& Hazleton, 1991; Mavis, Henry, Ogle \& Hoppe, 1996). These authors argue that OSCE fragments holistic patient care into discrete and unrelated elements as the students move between stations that presents unrelated scenarios. Bujack et al. (1991) advocate a model which uses a scenario-based examination to assess history-taking, physical examination, investigation ordering, interpretation of findings, diagnosis, management plan and communication within a single simulated patient.

The simulated assessment with random allocation refers to a situation where the students are randomly allocated patients/scenarios. This may be individually or in small groups and students are to examine the allocated patient/scenario and come up with findings. The counter point in this is that students are assessed on different scenarios, even though the assessment is objective

\section{Preparation and planning for OSCE}

The preparation and planning for OSCE involves the students, the assessors, the venue and the simulated patients. The students, the assessors and the patients should undergo a session on orientation for OSCE. The orientation could be done through a power-point or video presentation or a trial run on a similar platform.

- Preparation of the students

The students should be informed about the number of stations and the duration of each station and that there will be information at each station regarding the expected role, which information should be read carefully before the activity/procedure/response is undertaken. The student should also be informed about the time-keeper, the movement through the stations as well as the presence and the role of the assessor/s at all the stations.

- Preparation of the assessors and the assessment tools

The assessors should be oriented with regard to the assessment process, the tools that will be used at particular stations, the duration at each station and the information that the assessor and student should look out for in the stations. The assessment tools should be tested before being used in order to ensure validity. The tools should be developed in a similar format to ensure scoring reliability (Khan et al., (2013). 
- Preparation of the patient

Live patients participating in the OSCE are trained on how to behave during an assessment. The facilitators have to guide them on how to respond in their different stations depending on what is to be assessed. In many instances low fidelity mannequins are used whereupon the necessary equipment has to be assembled.

- Preparation of the venue

The venue should be able to accommodate the students and the stations that need to be assessed. Movement of students should be free-flowing and preferably clockwise from one station to the next. There should be privacy to enable the students to communicate freely without being seen and heard by the other students who might be at other stations.

\section{Scoring rubric}

Scoring methods for OSCEs also vary widely and are open to debate. The design and choice of tools used to score students' performance are key aspects of reliability and validity. A checklist is commonly used as a scoring method during OSCE. Scoring rubrics require examiners to tick each element from a list as either 'done' or 'not done'. Stevens and Levi (2005) referred to a scoring rubric as an assessment tool or instrument that delineates the expectations for a task or an assignment. These rubrics are sometimes referred to as criterion rating tools. Khan et al., (2013) identify two main types of scoring rubrics, namely analytical and holistic.

\section{Analytical scoring (checklist scale)}

A checklist is a series of statements describing the actions expected of candidates at the station. It is prepared in advance, following consultation with the team designing the OSCE stations and in line with the content and outcomes being assessed. Checklists could be 'binary', yes/no (performed/not performed). Alternatively, checklists can have a 3-5 point rating scale, which allows the examiners to mark candidates based upon the quality of their actions (Khan et al., 2013). A majority of researchers have raised concerns regarding the use of checklists in that they undermine the examiner's expert clinical judgement (Miller et al. 1998; Bartfay et al., 2004). This has in turn resulted in a number of adaptations such as the 
inclusion of elements of global rating (holistic rating) by differentiating the relative quality of students' performance.

\section{Holistic scoring (global rating scale)}

Global rating scales allow the assessor to rate the whole process in order to accurately reflect the skill of the candidate. Global scales allow examiners to determine not only whether an action was performed, but also how well it was performed. This tool is therefore better for assessing skills where the quality of performance is as important as the action itself. An example might be the nurse-patient relationship during the assessment. Hence, holistic scales are more useful for assessing areas such as judgement, empathy, organisation of knowledge and technical skills (Morgan et al., 2001; Hodges \& McIlroy, 2003).

\section{Pass mark}

Another issue that affects the reliability and validity of the scoring rubric is the 'pass mark'. A key consideration is the level of competence required for the particular professional role being assessed (Rushforth, 2007). In the UK, 40\% is a pass mark for undergraduate assessment and 50\% for Master's programme assessment. This is especially difficult in situations where marks are largely based on the checklist approach. It is, arguably, unacceptable for a student to be regarded as safe to practise if they have omitted or poorly performed with regard to $60 \%$ of the required elements. Use of negative marking or highlighted 'compulsory' or 'red flag' symbols have been used by some institutions to address this concern, whereupon the omission of these safety related items: for example, failure to identify a patient before administering a drug would lead to the student automatically failing, irrespective of the percentage achieved.

\section{Implementing the OSCE}

The success in the implementation of OSCE is based on its preparation and planning. Briefing of learners, examiners and simulated patients should be undertaken separately on the day of the examination. The movement of learners from one station to another can be managed by use of a bell coupled with verbal instructions, such as 'start' and 'stop', with the aid of a sound system. The learners should be warned about time, such as receiving an indication that they have two minutes left to complete a particular skill. Staff members should 
be available to make sure that the learners move in the correct direction. If there is a need to replace equipment or simulated patients, this should be done promptly to avoid confusion in the flow and unnecessary delay that may prolong the duration of the OSCE (Khan et al., 2013).

The assessors should remain at the allocated stations and use the assessment tools as required. Admission into the OSCE venue must be managed similarly to all assessment venue access. The learner's identity should be checked and the attendance register should also be signed by all the learners who are coming in to be assessed. During OSCE there should be minimal communication between the assessor/s and the learners as this might divert the learner's focus from the task at hand. The learner should communicate with the simulated patient and avoid focusing on the assessors' recording on the assessment tool.

The simulated patients should ask questions as highlighted during the orientation session and, to enable objective assessment. They should avoid changing their behaviour. Should the simulated patient feel tired, the replacement should be done promptly to avoid interruption of the planned assessment period. If there is a resting station, the learners should be informed about this as one is likely to start the cycle at such a station.

\section{Quality assurance strategies}

The quality assurance of each examination is a continuous process repeated with each examination cycle. Although many quality assurance procedures take place in the OSCE, quality assurance is also by training facilitators on how to conduct assessments, peer reviewing of questions to be asked and setting of OSCE stations and ensuring standardisation in all the processes before commencement of the examination. Kahn et al. (2013) identify the following elements of OSCE quality assurance: external examiners involvement, examiner training, peer review and standardisation of assessment tools. For example, these authors recommend that external examiners may be invited from different institutions to ensure that the assessment process measures student achievement rigorously and fairly and is conducted in line with policies and regulations. Assessment tools to be moderated internally and externally. 
The feedback on the examination process provided by the examiners can be used to improve the quality of the assessment tool and stations and the organisation of future examinations. Students may also be invited to provide feedback on their experiences as part of the quality assurance process (Kahn et al., 2013). The assessment policy of the institution should be checked for alignment with the OSCE. The assessment tools should also be designed in accordance with the curriculum and assessment plan. The information given to the assessors, students and simulated patients should also be monitored for consistency. These measures will ensure that all students are exposed to similar conditions at all the stations.

\section{Advantages of OSCE}

OSCE provides for a meaningful alternative strategy as it allows for individual assessments of a total group or class of students in a timely, controlled and safe manner. In this sense, student's competence can be assessed with minimal risk to patients (Mitchell, Henderson, Groves, Dalton \& Nulty, 2009). A study by Barry, Noonan, Bradshaw \& Murphy-Tighe, (2012) revealed that student midwives perceived OSCE as a valid means of assessment and that it increased confidence in performing clinical skills. OSCEs are seen as valid and reliable tools for assessment with explicit criteria to assess knowledge and skills (Barry et al., 2012). The OSCE method is more objective than most other assessment forms and tests a broader range of skills.

OSCE as a means of assessment can also provide the student with confidence when faced with challenges in practice. OSCE has been praised for allowing students to develop and improve their clinical skills within a controlled environment (Muldoon, Briesty \& Smith, 2013). OSCE encourages students to enhance their own learning and reflection in a safe environment (Rushforth, 2006).

An advantage of using OSCE as a means of assessment is that it provides a standardised assessment tool, enabling a higher level of inter-observer reliability over a range of examiners and students, as all students are assessed by the same criteria, thus reducing subjectivity. As the assessment is standardised, marking variability is reduced between examiners, leading to more objectivity and less marker interpretation. A further advantage of OSCE as a form of assessment is that it offers a setting where the environment is safe and practice can be simulated (Clarke, Rainey \& Traynor, 2011). Major (2005) points out that the simulated 
environment can be controlled according to the level of skill and the learning needs of the student. Another advantage of using the OSCE is the flexibility of its components. It can take the form of small scenarios, simulations, case studies, multiple choice questions or even rest stations to help the students relax in between the stations (Alinier, 2003). An OSCE provides students with an opportunity to demonstrate clinical skills and offers them a forum for rectifying errors without putting patients at risk (Taras, 2005). In support of the OSCE, 95\% of nursing students in Riyadh, Saudi Arabia, agreed that OSCE was a realistic assessment for nursing courses; in relation to quality, 95\% said it was fair, 90\% stated that it covered a wide range of knowledge and $96 \%$ appreciated the manner in which it was administered (Eswi et al, 2013).

\section{Disadvantages of OSCE}

Muldoon, Briesty \& Smith (2013) indicate that the majority of midwifery students expressed that they felt very nervous during the OSCE. Nervousness, stress and anxiety are likely to be associated with any examination procedure. According to students, stress was experienced, especially prior to entering the assessment room (Mårtensson \& Löfmark, 2013). Clarke, Rainey \& Traynor, (2011) reported that students showed a tendency to speak to the examiner rather than the simulated patient during the OSCE.

During the OSCE there is a risk of student and examiner fatigue (Harden \& Gleeson, 1979; Major, 2005). Examiner fatigue is caused by the number of students who pass through each station, as well as the concentration required in watching the same skill being repeated on many occasions. Student fatigue may occur due to concentrating on performing the clinical skills within an intense examination environment in a short space of time (Clarke, Rainey \& Traynor, 2011). Chabeli (2001) further states that lack of human and material resources may be considered as a limitation in this form of assessment. It can be frustrating for students and examiners when they have to move from one department to another requesting equipment and in some instance finally having to simulate even the equipment.

\section{Conclusion}

Accurate assessment of clinical competence is vital. It is concluded that the OSCE is a valuable, fair and practical method of assessing clinical competence in nursing students. However, successful OSCE is often the result of significant planning, co-ordination of 
resources and adhering to quality assurance strategies. Proper management and awareness of potential problems make the OSCE a successful method for assessing clinical competence.

\section{References}

Alinier, G. 2003. Nursing students' and lecturers' perspectives of objective structured clinical examination incorporating simulation. Nurse Education Today 23: 419-426.

Barry, M., Noonan, M., Bradshaw, C. \& Murphy-Tighe, S. 2012. An exploration of student midwives of the Objective Structured Clinical Examination. Nurse Education Today 32: 690-694.

Benner, P. 1982. From novice to expert. American Journal of Nursing 82 (3): 402-407.

1984. From novice to expert: Excellence and power in nursing practice. Menlo Park: Addison-Wesley.

Bujack, L., McMillan, M., Dwyer, J. \& Hazleton, M. 1991. Assessing comprehensive nursing performance: the objective structured clinical assessment (OSCA). Part 1: development of the assessment strategy. Nursing Education Today 11: 179-184.

Chabeli, M.M. 2001. Nurse educators' perceptions of OSCE as a clinical evaluation method. Curationis 24 (1): 84-92.

Clarke, S., Rainey, D. \& Traynor, M. 2011. Using Objective Structured Clinical Examination. International Journal of Orthopaedic and Trauma Nursing (15): 92-101.

Ebbert, D. \& Connors, H. 2004. Standardised patient experiences: Evaluation of clinical performance and nurse practitioner student satisfaction. Nursing Education Perspectives $1: 12-15$.

Eswi, A., Samy, A., Shaliabe, H. 2013. OSCE in maternity and community health nursing: Saudi nursing students' perspective. American Journal of Research and Communication. Vol1(3),143-162 
Frantz,, J.M., Rowe, M., Hess, D.A., Rhoda, A.J., Sauls, B.L. \& Wegner, L. 2013. Student and staff perceptions and experiences of the introduction of Objective Structured Practical Examinations: A pilot study. African Journal of Health Professions Education 5 (2): 72-74.

Gibbons, S., Adamo, G., Padden, D., Ricciardi, R., Graziano, M., Levine, N., 2002. Clinical evaluation in advanced practice nursing education: Using standardised patients in health assessment. Journal of Nursing Education 41: 215-221.

Harden, R.M. \& Gleeson, F.A. 1979. Assessment of clinical competence using an objective Structured Clinical Examination (OSCE). British Medical Journal 13 (1):41-54.

Harden, R.M., Stevenson, M., Downie, W.W., and Wilson G. M 1975. Assessment of clinical competence using objective structured clinical examination. British Medical Journal 1: 447-451.

Hodges, B. \& McIlroy, J.H. 2003. Analytic global OSCE ratings are sensitive to level of training. Medical Education 37: 1012-1016.

Khan, K.Z.,Gaunt, K., Ramachandran, S. \& Pushkar, P. 2013. The objective structured clinical examination (OSCE): AMEE Guide No. 81. Part II: Organisation \& administration. Medical Teacher 35: e1447-e1463.

Khattab, A. \& Rawlings, B. 2001. Assessing nurse practitioner students using a modified objective structured clinical examination (OSCE). Nurse Education Today 21: 541-550.

Kurz J.M., Mahoney, K., Martin-Plank, L. \& Lidicker, J. 2009. Objective structured clinical examination and advanced practice nursing students. Journal of Professional Nursing 25 (3): 186-191.

Major, D.A. 2005. OSCEs-Seven years on the bandwagon: The progress of an Objective Structured Clinical Evaluation programme. Nurse Education Today 25 (6):442-454. 
Mårtensson, G. \& Löfmark, A. 2013. Implementation and student evaluation of clinical final examination in nursing education. Nurse Education Today 33: 1563-1568.

Mavis, B., Henry, R., Ogle, K. \& Hoppe, R. 1996. The emperor's new clothes: the OSCE revisited. Academic Medicine 71 (5): 447-453.

Meyer, S.M. \& van Niekerk, S.E. 2008. Nurse educator in practice. Cape Town: Juta.

Mitchell, M.L., Henderson, A., Groves, M., Dalton, N., Nulty, D. 2009. The objective structured clinical examination (OSCE): optimising its value in the undergraduate nursing curriculum. Nurse Education Today 30: 345-348.

Morgan, P.J., Cleave-Hogg, D. \& Guest, C.B. 2001. A comparison of global ratings and checklist scores from an undergraduate assessment using an anaesthesia simulator. Academic Medical 76: 1053-1055.

Muldoon, K., Biesty, L. \& Smith, V. 2013. 'I found the OSCE very stressful: Student midwives' attitudes towards an objective structured clinical examination (OSCE). Nurse Education Today 34 (3): 468-473.

Rushforth, H.E. 2007. OSCE: review of literature and implications for nursing education. Nurse Education Today 27: 481-490.

Smith, V., Muldoon, K. \& Biesty, L. 2012. The objective structured clinical examination (OSCE) as a strategy for assessing clinical competence in midwifery education in Ireland: A critical review. Nursing Education in Practice 12: 242-247.

Stevens, D.D. \& Levi, A. 2005. Introduction to rubrics: An assessment tool to save grading time, convey effective feedback and promote student learning. Sterling, VA: Stylus Publishers.

Taras, M. 2005. Assessment-summative and formative-some theoretical reflections. British Journal of Educational Studies 53 (4): 466-478. 
Turner, J.L. \& Dankoski, M.E. 2008. Objective structured clinical exams: A critical review. Family Medicine 40 (8): 574-578.

Ward, H. \& Barratt, J. 2005. Assessment of nurse practitioner advanced clinical practice skills: using the objective structured clinical examination (OSCE). Primary Health Care 16 (3): $22-24$.

Ward, H. \& Willis, A. 2006. Assessing advanced clinical practice skills. Primary Health Care 16 (3): 22-24. 